\title{
COVID-19 and BCG: where's the challenge?
}

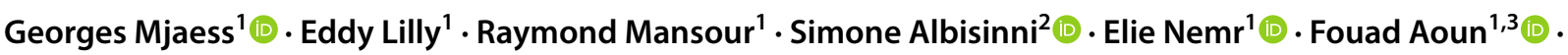 \\ Thierry Roumeguere ${ }^{2,3}$ (])
}

Received: 30 November 2020 / Accepted: 5 December 2020 / Published online: 2 January 2021

(c) The Author(s), under exclusive licence to Springer-Verlag GmbH, DE part of Springer Nature 2021

Keywords COVID-19 $\cdot$ BCG $\cdot$ Urology $\cdot$ BCGitis $\cdot$ Algorithm

Dear Editor,

The COVID-19 pandemic has a huge impact on urological practice. Due to benefit on respiratory infections, Bacillus Calmette-Guerin (BCG) vaccination programs have been proposed to decrease COVID-19 infection severity, and this is due to its stimulatory effect on immunity. Prospective randomized controlled trials are ongoing to reveal this matter [1]. Desouky has discussed in his review the use of BCG in the context of COVID-19 and its potential additional impact on BCG shortage, especially in the urological field [2].

However, recent strong evidence also suggested that receiving the BCG vaccine in the past does not have a protective effect against COVID-19 [3]. Moreover, numerous specific vaccines are emerging for COVID-19 and seem to have promising results. Therefore, the idea of the impact of using BCG vaccination in the context of COVID-19 prevention could become irrational at present.

Nevertheless, another riddling challenge that we are often facing in our clinical practice is overlapping clinical

Georges Mjaess and Eddy Lilly contributed equally to this manuscript.

This comment refers to the article available online at https://doi. org/10.1007/s00345-020-03251-7.

Georges Mjaess

gmjaess@gmail.com; georges.mjaess@net.usj.edu.lb

1 Department of Urology, Hotel-Dieu de France, Beirut, Lebanon

2 Department of Urology, University Clinics of Brussels, Erasme Hospital, Université Libre de Bruxelles, Brussels, Belgium

3 Department of Urology, Jules Bordet Institute, Brussels, Belgium presentation between the infectious complications of BCG intravesical instillation and COVID-19 infection.

From a clinical point of view, moderate fever is a common symptom after BCG intravesical instillation [4]. A more severe fever, higher than $38.5^{\circ} \mathrm{C}$, and lasting more than $72 \mathrm{~h}$ is associated with locoregional and systemic disseminated BCG, which represent $1 \%$ and $4.3 \%$ of BCGitis cases, respectively [4]. Therefore, in systemic disseminated BCG, fever is almost persistent, with sweating, flu-like syndrome with dry cough, and malaise [5]. Pulmonary disease remains the most common presentation of systemic BCGitis [5]. All these clinical findings are common for COVID-19 patients.

Radiologic assessment by chest CT can sometimes be misleading in the differential diagnosis. In BCGitis, it shows typically miliary disease, but around $25 \%$ of patients will present with reticulonodular interstitial infiltrates [5]. In COVID-19 patients, the most common radiographic findings are ground glass opacities, consolidations, and/or reticulonodular interstitial infiltrates [6]. Differentiating between these two diseases on chest CT can therefore be challenging.

Moreover, bronchoalveolar lavage in both cases will show alveolar lymphocytic infiltrate with predominant T-CD4 [7]. The low sensitivity (42\%) of the mycobacterium tuberculosis complex PCR test done on biopsies or cultures approves the inflammatory response theory [8].

These similar findings are due to comparable pathophysiology of both diseases. In fact, in both cases it is the inflammatory response (hypersensitivity and cytokine storm) that plays the important role rather than the responsible pathogen itself $[9,10]$. On the one hand, attributing a COVID-19 case to adverse effects of intravesical BCG therapy holds an important risk of viral dissemination to urologists and patient surroundings, especially that a negative PCR test for COVID-19 does not rule out the diagnosis since it holds a significant number of false-negative results. On the other hand, attributing serious BCGitis to COVID-19 could 


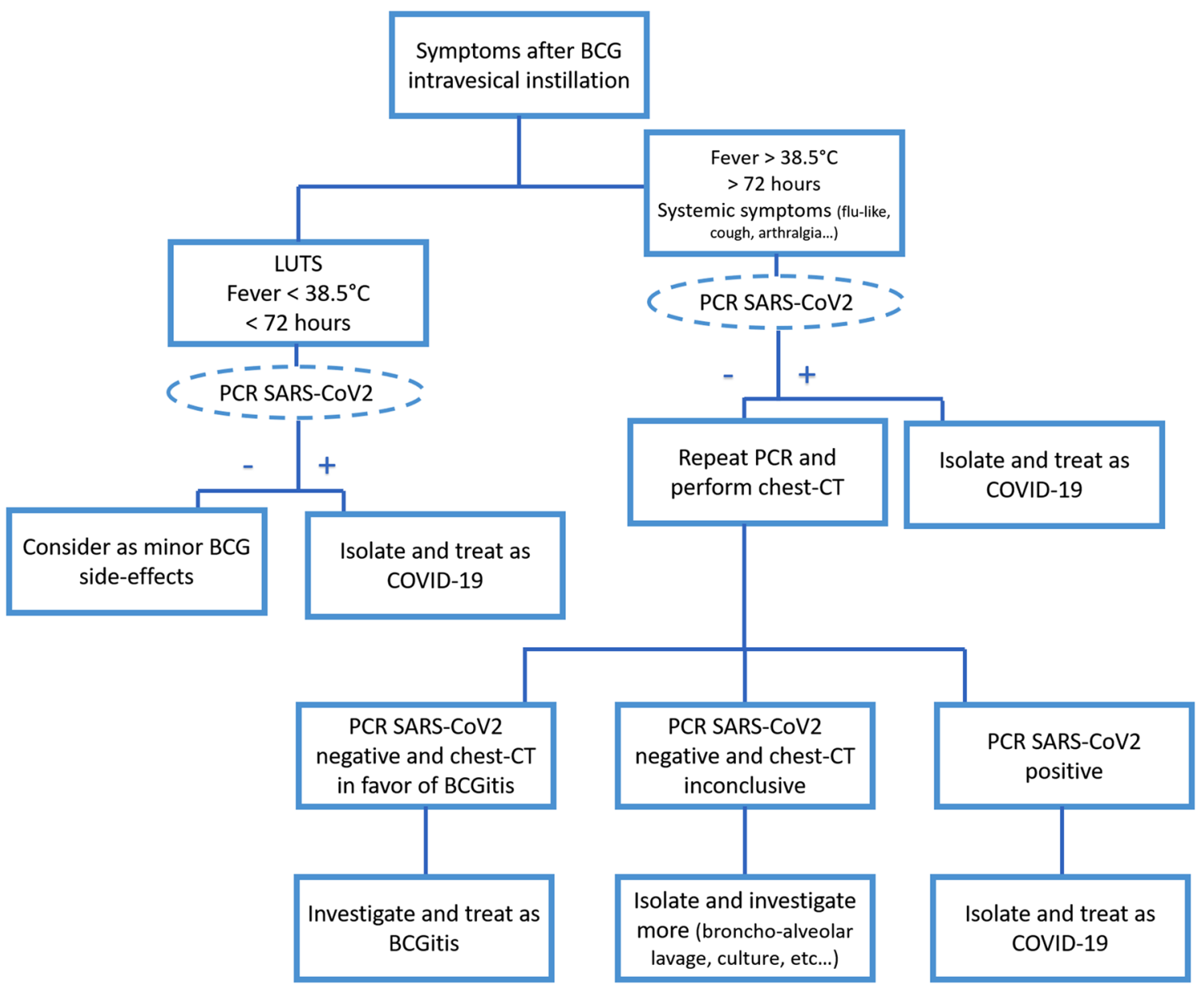

Fig.1 Diagnostic algorithm of infectious symptoms in patients receiving intravesical BCG instillations in the COVID-19 era

impede and mislead the diagnosis of BCGitis which could be fatal in a significant number of cases.

We have proposed a new algorithm to manage these overlapping clinical features in patients receiving intravesical BCG instillations (Fig. 1).

To conclude, COVID-19 should remain a crucial differential diagnosis in bladder cancer patients treated with intravesical BCG instillations, especially that BCGitis remains a rare complication.

Author contributions GM and EL performed conceptualization; GM, EL and RM were involved in writing-manuscript draft; SA, EN, FA and TR writing - review and editing. TR done a supervision.

Funding This paper did not receive any funding.

\section{Compliance with ethical standards}

Conflict of interest All the authors have no conflict of interest to disclose.

\section{References}

1. Brooks NA, Narayan V, Hegarty PK, Zafirakis H, Han X, Kamat AM (2020) The role of the urologist, BCG vaccine administration, and SARS-CoV-2: an overview. Bjui Compass 1(3):87-92

2. Desouky E (2020) BCG versus COVID-19: impact on urology. World J Urol 16(16):1-5. https://doi.org/10.1007/s00345-02003251-7

3. de Chaisemartin C, de Chaisemartin L. BCG vaccination in infancy does not protect against COVID-19. Evidence from a natural experiment in Sweden. Clin Infect Dis Off Publ Infect Dis Soc Am [Internet]. 2020 Aug 23 [cited 2020 Nov 29]; Available from: https://www.ncbi.nlm.nih.gov/pmc/articles/ PMC7499491/

4. Pérez-Jacoiste Asín MA, Fernández-Ruiz M, López-Medrano F, Lumbreras C, Tejido A, San Juan R et al (2014) Bacillus Calmette-Guérin (BCG) infection following intravesical BCG administration as adjunctive therapy for bladder cancer: incidence, risk factors, and outcome in a single-institution series and review of the literature. Medicine (Baltimore) 93(17):236-254

5. Levi L, Groh M, De Castro N, Bergeron A, Schlemmer F (2018) BCG infection following intravesicular immunotherapy for bladder cancer. Rev Mal Respir 35(4):416-429 
6. Cascella M, Rajnik M, Cuomo A, Dulebohn SC, Di Napoli R. Features, Evaluation, and Treatment of Coronavirus. In: StatPearls [Internet]. Treasure Island (FL): StatPearls Publishing; 2020 [cited 2020 Nov 28]. Available from: http://www.ncbi. nlm.nih.gov/books/NBK554776/

7. Voiriot G, Fajac A, Lopinto J, Labbé V, Fartoukh M (2020) Bronchoalveolar lavage findings in severe COVID-19 pneumonia. Intern Emerg Med 15:1-2

8. Larsen BT, Smith ML, Grys TE, Vikram HR, Colby TV (2015) Histopathology of disseminated mycobacterium bovis infection complicating intravesical BCG immunotherapy for urothelial carcinoma. Int J Surg Pathol 23(3):189-195

9. Fu L, Wang B, Yuan T, Chen X, Ao Y, Fitzpatrick T et al (2020) Clinical characteristics of coronavirus disease 2019 (COVID-19) in China: a systematic review and meta-analysis. J Infect 80(6):656-665

10. Um S-J, Lee S-K, Yang D-K (2009) Hypersensitivity pneumonitis following intravesical bacille Calmette-Guérin immunotherapy for superficial bladder cancer. J Investig Allergol Clin Immunol 19(3):230-232

Publisher's Note Springer Nature remains neutral with regard to jurisdictional claims in published maps and institutional affiliations. 